\title{
Packing Plane Spanning Trees into a Point Set
}

\author{
Ahmad Biniaz*
}

Alfredo García ${ }^{\dagger}$

\begin{abstract}
Let $P$ be a set of $n$ points in the plane in general position. We show that at least $\lfloor n / 3\rfloor$ plane spanning trees can be packed into the complete geometric graph on $P$. This improves the previous best known lower bound $\Omega(\sqrt{n})$. Towards our proof of this lower bound we show that the center of a set of points, in the $d$-dimensional space in general position, is of dimension either 0 or $d$.
\end{abstract}

\section{Introduction}

In the two-dimensional space, a geometric graph $G$ is a graph whose vertices are points in the plane and whose edges are straight-line segments connecting the points. A subgraph $S$ of $G$ is plane if no pair of its edges cross each other. Two subgraphs $S_{1}$ and $S_{2}$ of $G$ are edge-disjoint if they do not share any edge.

Let $P$ be a set of $n$ points in the plane. The complete geometric graph $K(P)$ is the geometric graph with vertex set $P$ that has a straight-line edge between every pair of points in $P$. We say that a sequence $S_{1}, S_{2}, S_{3}, \ldots$ of subgraphs of $K(P)$ is packed into $K(P)$, if the subgraphs in this sequence are pairwise edge-disjoint. In a packing problem, we ask for the largest number of subgraphs of a given type that can be packed into $K(P)$. Among all subgraphs, plane spanning trees, plane Hamiltonian paths, and plane perfect matchings are of interest. Since $K(P)$ has $n(n-1) / 2$ edges, at most $\lfloor n / 2\rfloor$ spanning trees, at most $\lfloor n / 2\rfloor$ Hamiltonian paths, and at most $n-1$ perfect matchings can be packed into it.

A long-standing open question is to determine whether or not it is possible to pack $\lfloor n / 2\rfloor$ plane spanning trees into $K(P)$. If $P$ is in convex position, the answer in the affirmative follows from the result of Bernhart and Kanien [3], and a characterization of such plane spanning trees is given by Bose et al. [5]. In CCCG 2014, Aichholzer et al. [1] showed that if $P$ is in general position (no three points on a line), then $\Omega(\sqrt{n})$ plane spanning trees can be packed into $K(P)$; this bound is obtained by a clever combination of crossing family (a set of pairwise crossing edges) [2] and double-stars (trees with only two interior nodes) [5]. Schnider [12] showed that it is not always possible to pack $\lfloor n / 2\rfloor$ plane spanning double stars into $K(P)$, and gave a necessary and sufficient condition for the existence of such a packing. As for packing other spanning structures into $K(P)$, Aichholzer et al. [1] and Biniaz et al. [4] showed a packing of 2 plane Hamiltonian cycles and a packing of $\left\lceil\log _{2} n\right\rceil-2$ plane perfect matchings, respectively.

The problem of packing spanning trees into (abstract) graphs is studied by Nash-Williams [11] and Tutte [13] who independently obtained necessary and sufficient conditions to pack $k$ spanning trees into a graph. Kundu [10] showed that at least $\lceil(k-1) / 2\rceil$ spanning trees can be packed into any $k$-edge-connected graph.

In this paper we show how to pack $\lfloor n / 3\rfloor$ plane spanning trees into $K(P)$ when $P$ is in general position. This improves the previous $\Omega(\sqrt{n})$ lower bound.

\footnotetext{
*University of Waterloo, Canada. Supported by NSERC Postdoctoral Fellowship. ahmad.biniaz@gmail.com

${ }^{\dagger}$ Universidad de Zaragoza, Spain. Partially supported by H2020-MSCA-RISE project 734922 - CONNECT and MINECO project MTM2015-63791-R. olaverri@unizar.es
} 


\section{Packing Plane Spanning Trees}

In this section we show how to pack $\lfloor n / 3\rfloor$ plane spanning tree into $K(P)$, where $P$ is a set of $n \geqslant 3$ points in the plane in general position (no three points on a line). If $n \in\{3,4,5\}$ then one can easily find a plane spanning tree on $P$. Thus, we may assume that $n \geqslant 6$.

The center of $P$ is a subset $C$ of the plane such that any closed halfplane intersecting $C$ contains at least $\lceil n / 3\rceil$ points of $P$. A centerpoint of $P$ is a member of $C$, which does not necessarily belong to $P$. Thus, any halfplane that contains a centerpoint, has at least $\lceil n / 3\rceil$ points of $P$. It is well known that every point set in the plane has a centerpoint; see e.g. [7, Chapter 4]. We use the following corollary and lemma in our proof of the $\lfloor n / 3\rfloor$ lower bound; the corollary follows from Theorem 3 that we will prove later in Section 3.

Corollary 1. Let $P$ be a set of $n \geqslant 6$ points in the plane in general position, and let $C$ be the center of $P$. Then, $C$ is either 2-dimensional or 0-dimensional. If $C$ is 0 -dimensional, then it consists of one point that belongs to $P$, moreover $n$ is of the form $3 k+1$ for some integer $k \geqslant 2$.

Lemma 1. Let $P$ be a set of $n$ points in the plane in general position, and let $c$ be a centerpoint of $P$. Then, for every point $p \in P$, each of the two closed halfplanes, that are determined by the line through $c$ and $p$, contains at least $\lceil n / 3\rceil+1$ points of $P$.

Proof. For the sake of contradiction assume that a closed halfplane $\bar{H}$, that is determined by the line through $c$ and $p$, contains less than $\lceil n / 3\rceil+1$ points of $P$. By symmetry assume that $\bar{H}$ is to the left side of this line oriented from $c$ to $p$; see the figure to the right. Since $c$ is a centerpoint and $\bar{H}$ contains $c$, the definition of centerpoint implies that $\bar{H}$ contains exactly $\lceil n / 3\rceil$ points of $P$ (including $p$ and any other point of $P$ that may lie on the boundary of $\bar{H})$. By slightly rotating $\bar{H}$ counterclockwise around $c$, while keeping $c$ on the boundary of $\bar{H}$, we obtain a new closed halfplane that contains $c$ but

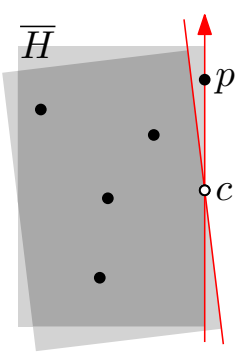
misses $p$. This new halfplane contains less than $\lceil n / 3\rceil$ points of $P$; this contradicts $c$ being a centerpoint of $P$.

Now we proceed with our proof of the lower bound. We distinguish between two cases depending on whether the center $C$ of $P$ is 2-dimensional or 0-dimensional. First suppose that $C$ is 2-dimensional. Then, $C$ contains a centerpoint, say $c$, that does not belong to $P$. Let $p_{1}, \ldots, p_{n}$ be a counter-clockwise radial ordering of points in $P$ around $c$. For two points $p$ and $q$ in the plane, we denote by $\overrightarrow{p q}$, the ray emanating from $p$ that passes through $q$.

Since every integer $n \geqslant 3$ has one of the forms $3 k, 3 k+1$, and $3 k+2$, for some $k \geqslant 1$, we will consider three cases. In each case, we show how to construct $k$ plane spanning directed graphs $G_{1}, \ldots, G_{k}$ that are edge-disjoint. Then, for every $i \in\{1, \ldots, k\}$, we obtain a plane spanning tree $T_{i}$ from $G_{i}$. First assume that $n=3 k$. To build $G_{i}$, connect $p_{i}$ by outgoing edges to $p_{i+1}, p_{i+2}, \ldots, p_{i+k}$, then connect $p_{i+k}$ by outgoing edges to $p_{i+k+1}, p_{i+k+2}, \ldots, p_{i+2 k}$, and then connect $p_{i+2 k}$ by outgoing edges to $p_{i+2 k+1}, p_{i+2 k+2}, \ldots, p_{i+3 k}$, where all the indices are modulo $n$, and thus $p_{i+3 k}=p_{i}$. The graph $G_{i}$, that is obtained this way, has one cycle $\left(p_{i}, p_{i+k}, p_{i+2 k}, p_{i}\right)$; see Figure 1. By Lemma 1, every closed halfplane, that is determined by the line through $c$ and a point of $P$, contains at least $k+1$ points of $P$. Thus, all points $p_{i}, p_{i+1}, \ldots, p_{i+k}$ lie in the closed halfplane to the left of the line through $c$ and $p_{i}$ that is oriented from $c$ to $p_{i}$. Similarly, the points $p_{i+k}, \ldots, p_{i+2 k}$ lie in the closed halfplane to the left of the oriented line from $c$ to $p_{i+k}$, and the points $p_{i+2 k}, \ldots, p_{i+3 k}$ lie in the closed halfplane to the left of the oriented line from $c$ to $p_{i+2 k}$. Thus, all the $k$ edges outgoing from $p_{i}$ are in the convex wedge bounded by the rays $\overrightarrow{c p_{i}}$ and $\overrightarrow{c p_{i+k}}$, all the edges outgoing from $p_{i+k}$ are in the convex wedge bounded by $\overrightarrow{c p_{i+k}}$ and $\overrightarrow{c_{i+2 k}}$, and all the edges from $p_{i+2 k}$ are in the convex wedge bounded by $\overrightarrow{c p_{i+2 k}}$ and $\overrightarrow{c_{i+3 k}}$. 
Therefore, the spanning directed graph $G_{i}$ is plane. As depicted in Figure 1, by removing the edge $\left(p_{i+2 k}, p_{i}\right)$ from $G_{i}$ we obtain a plane spanning (directed) tree $T_{i}$. This is the end of our construction of $k$ plane spanning trees.
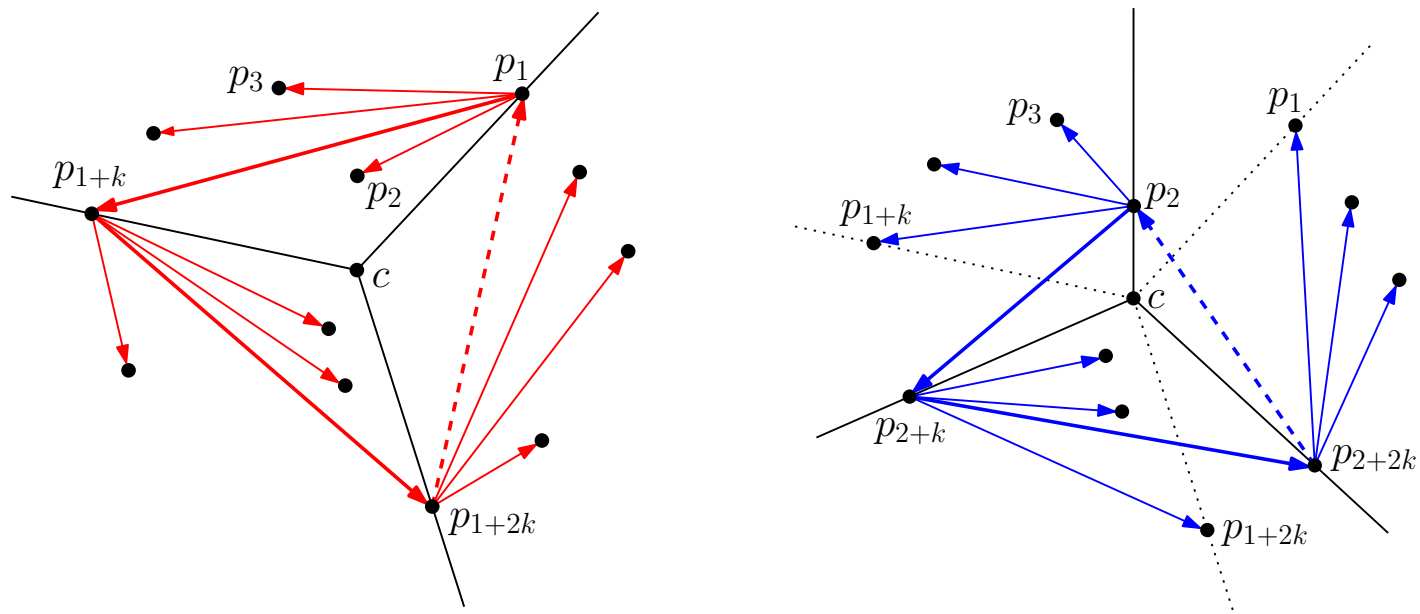

Figure 1: The plane spanning trees $T_{1}$ (the left) and $T_{2}$ (the right) are obtained by removing the edges $\left(p_{1+2 k}, p_{1}\right)$ and $\left(p_{2+2 k}, p_{2}\right)$ from $G_{1}$ and $G_{2}$, respectively.

To verify that the $k$ spanning trees obtained above are edge-disjoint, we show that two trees $T_{i}$ and $T_{j}$, with $i \neq j$, do not share any edge. Notice that the tail of every edge in $T_{i}$ belongs to the set $I=\left\{p_{i}, p_{i+k}, p_{i+2 k}\right\}$, and the tail of every edge in $T_{j}$ belongs to the set $J=\left\{p_{j}, p_{j+k}, p_{j+2 k}\right\}$, and $I \cap J=\emptyset$. For contrary, suppose that some edge $\left(p_{r}, p_{s}\right)$ belongs to both $T_{i}$ and $T_{j}$, and without loss of generality assume that in $T_{i}$ this edge is oriented from $p_{r}$ to $p_{s}$ while in $T_{j}$ it is oriented from $p_{s}$ to $p_{r}$. Then $p_{r} \in I$ and $p_{s} \in J$. Since $\left(p_{r}, p_{s}\right) \in T_{i}$ and the largest index of the head of every outgoing edge from $p_{r}$ is $r+k$, we have that $s \leqslant(r+k)$ $\bmod n$. Similarly, since $\left(p_{s}, p_{r}\right) \in T_{j}$ and the largest index of the head of every outgoing edge from $p_{s}$ is $s+k$, we have that $r \leqslant(s+k) \bmod n$. However, these two inequalities cannot hold together; this contradicts our assumption that $\left(p_{r}, p_{s}\right)$ belongs to both trees. Thus, our claim, that $T_{1}, \ldots, T_{k}$ are edge-disjoint, follows. This finishes our proof for the case where $n=3 k$.

If $n=3 k+1$, then by Lemma 1 , every closed halfplane that is determined by the line through $c$ and a point of $P$ contains at least $k+2$ points of $P$. In this case, we construct $G_{i}$ by connecting $p_{i}$ to its following $k+1$ points, i.e., $p_{i+1}, \ldots, p_{i+k+1}$, and then connecting each of $p_{i+k+1}$ and $p_{i+2 k+1}$ to their following $k$ points. If $n=3 k+2$, then we construct $G_{i}$ by connecting each of $p_{i}$ and $p_{i+k+1}$ to their following $k+1$ points, and then connecting $p_{i+2 k+2}$ to its following $k$ points. This is the end of our proof for the case where $C$ is 2-dimensional.

Now we consider the case where $C$ is 0 -dimensional. By Corollary $1, C$ consists of one point that belongs to $P$, and moreover $n=3 k+1$ for some $k \geqslant 2$. Let $p \in P$ be the only point of $C$, and let $p_{1}, \ldots, p_{n-1}$ be a counter-clockwise radial ordering of points in $P \backslash\{p\}$ around $p$. As in our first case (where $C$ was 2-dimensional, $c$ was not in $P$, and $n$ was of the form $3 k$ ) we construct $k$ edge-disjoint plane spanning trees $T_{1}, \ldots, T_{k}$ on $P \backslash\{p\}$ where $p$ playing the role of $c$. Then, for every $i \in\{1, \ldots, k\}$, by connecting $p$ to $p_{i}$, we obtain a plane spanning tree for $P$. These plane spanning trees are edge-disjoint. This is the end of our proof. In this section we have proved the following theorem.

Theorem 1. Every complete geometric graph, on a set of $n$ points in the plane in general position, contains at least $\lfloor n / 3\rfloor$ edge-disjoint plane spanning trees. 


\section{The Dimension of the Center of a Point Set}

The center of a set $P$ of $n \geqslant d+1$ points in $\mathbb{R}^{d}$ is a subset $C$ of $\mathbb{R}^{d}$ such that any closed halfspace intersecting $C$ contains at least $\alpha=\lceil n /(d+1)\rceil$ points of $P$. Based on this definition, one can characterize $C$ as the intersection of all closed halfspaces such that their complementary open halfspaces contain less than $\alpha$ points of $P$. More precisely (see [7, Chapter 4]) $C$ is the intersection of a finite set of closed halfspaces $\overline{H_{1}}, \overline{H_{2}}, \ldots, \overline{H_{m}}$ such that for each $\overline{H_{i}}$

1. the boundary of $\overline{H_{i}}$ contains at least $d$ affinely independent points of $P$, and

2. the complementary open halfspace $H_{i}$ contains at most $\alpha-1$ points of $P$, and the closure of $H_{i}$ contains at least $\alpha$ points of $P$.

Being the intersection of closed halfspaces, $C$ is a convex polyhedron. A centerpoint of $P$ is a member of $C$, which does not necessarily belong to $P$. It follows, from the definition of the center, that any halfspace containing a centerpoint has at least $\alpha$ points of $P$. It is well known that every point set in the plane has a centerpoint [7, Chapter 4]. In dimensions 2 and 3, a centerpoint can be computed in $O(n)$ time [9] and in $O\left(n^{2}\right)$ expected time [6], respectively.

A set of points in $\mathbb{R}^{d}$, with $d \geqslant 2$, is said to be in general position if no $k+2$ of them lie in a $k$-dimensional flat for every $k \in\{1, \ldots, d-1\} .{ }^{1}$ Alternatively, for a set of points in $\mathbb{R}^{d}$ to be in general position, it suffices that no $d+1$ of them lie on the same hyperplane. In this section we prove that if a point set $P$ in $\mathbb{R}^{d}$ is in general position, then the center of $P$ is of dimension either 0 or $d$. Our proof of this claim uses the following result of Grünbaum.

Theorem 2 (Grünbaum, $1962[8]$ ). Let $\mathcal{F}$ be a finite family of convex polyhedra in $\mathbb{R}^{d}$, let I be their intersection, and let $s$ be an integer in $\{1, \ldots, d\}$. If every intersection of $s$ members of $\mathcal{F}$ is of dimension $d$, but $I$ is $(d-s)$-dimensional, then there exist $s+1$ members of $\mathcal{F}$ such that their intersection is $(d-s)$-dimensional.
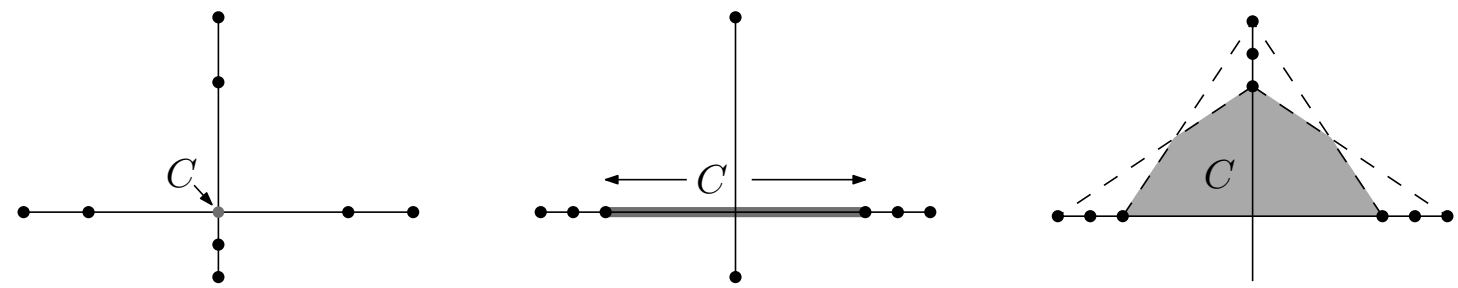

Figure 2: The dimension of a point set in the plane, that is not in general position, can be any number in $\{0,1,2\}$.

Before proceeding to our proof, we note that if $P$ is not in general position, then the dimension of $C$ can be any number in $\{0,1, \ldots, d\}$; see e.g. Figure 2 for the case where $d=2$.

Observation 1. For every $k \in\{1, \ldots, d+1\}$ the dimension of the intersection of every $k$ closed halfspaces in $\mathbb{R}^{d}$ is in the range $[d-k+1, d]$.

Theorem 3. Let $P$ be a set of $n \geqslant d+1$ points in $\mathbb{R}^{d}$, and let $C$ be the center of $P$. Then, $C$ is either d-dimensional, or contained in a $(d-s)$-dimensional polyhedron that has at least $n-(s+1)(\alpha-1)$ points of $P$ for some $s \in\{1, \ldots, d\}$ and $\alpha=\lceil n /(d+1)\rceil$. In the latter case if $P$ is in general position and $n \geqslant d+3$, then $C$ consists of one point that belongs to $P$, and $n$ is of the form $k(d+1)+1$ for some integer $k \geqslant 2$.

${ }^{1} \mathrm{~A}$ flat is a subset of $d$-dimensional space that is congruent to a Euclidean space of lower dimension. The flats in 2-dimensional space are points and lines, which have dimensions 0 and 1. 
Proof. The center $C$ is a convex polyhedron that is the intersection of a finite family $\mathcal{H}$ of closed halfspaces such that each of their complementary open halfspaces contains at most $\alpha-1$ points of $P\left[7\right.$, Chapter 4]. Since $C$ is a convex polyhedron in $\mathbb{R}^{d}$, its dimension is in the range $[0, d]$. For the rest of the proof we consider the following two cases.

(a) The intersection of every $d+1$ members of $\mathcal{H}$ is of dimension $d$.

(b) The intersection of some $d+1$ members of $\mathcal{H}$ is of dimension less than $d$.

First assume that we are in case (a). We prove that $C$ is $d$-dimensional. Our proof follows from Theorem 2 and a contrary argument. Assume that $C$ is not $d$-dimensional. Then, $C$ is $(d-s)$-dimensional for some $s \in\{1, \ldots, d\}$. Since the intersection of every $s$ members of $\mathcal{H}$ is $d$-dimensional, by Theorem 2 there exist $s+1$ members of $\mathcal{H}$ whose intersection is $(d-s)$ dimensional. This contradicts the assumption of case (a) that the intersection of every $d+1$ members of $\mathcal{H}$ is $d$-dimensional. Therefore, $C$ is $d$-dimensional in this case.

Now assume that we are in case (b). Let $s$ be the largest integer in $\{1, \ldots, d\}$ such that every intersection of $s$ members of $\mathcal{H}$ is $d$-dimensional; notice that such an integer exists because every single halfspace in $\mathcal{H}$ is $d$-dimensional. Our choice of $s$ implies the existence of a subfamily $\mathcal{H}^{\prime}$ of $s+1$ members of $\mathcal{H}$ whose intersection is $d^{\prime}$-dimensional for some $d^{\prime}<d$. Let $s^{\prime}$ be an integer such that $d^{\prime}=d-s^{\prime}$. By Observation 1 , we have that $d^{\prime} \geqslant d-s$, and equivalently $d-s^{\prime} \geqslant d-s$; this implies $s^{\prime} \leqslant s$. To this end we have a family $\mathcal{H}^{\prime}$ with $s+1$ members for which every intersection of $s^{\prime}$ members is $d$-dimensional (because $s^{\prime} \leqslant s$ and $\mathcal{H}^{\prime} \subseteq \mathcal{H}$ ), but the intersection of all members of $\mathcal{H}^{\prime}$ is $\left(d-s^{\prime}\right)$-dimensional. Applying Theorem 2 on $\mathcal{H}^{\prime}$ implies the existence of $s^{\prime}+1$ members of $\mathcal{H}^{\prime}$ whose intersection is $\left(d-s^{\prime}\right)$-dimensional. If $s^{\prime}<s$, then this implies the existence of $s^{\prime}+1 \leqslant s$ members of $\mathcal{H}^{\prime} \subseteq \mathcal{H}$, whose intersection is of dimension $d-s^{\prime}<d$. This contradicts the fact that the intersection of every $s$ members of $\mathcal{H}$ is $d$-dimensional. Thus, $s^{\prime}=s$, and consequently, $d^{\prime}=d-s^{\prime}=d-s$. Therefore $C$ is contained in a $(d-s)$-dimensional polyhedron $I$ which is the intersection of the $s+1$ closed halfspaces of $\mathcal{H}^{\prime}$. Let $H_{1}, \ldots, H_{s+1}$ be the complementary open halfspaces of members of $\mathcal{H}^{\prime}$, and recall that each $H_{i}$ contains at most $\alpha-1$ points of $P$. Let $\bar{I}$ be the complement of $I$. Then,

$$
\begin{aligned}
n & =|I \cup \bar{I}|=\left|I \cup H_{1} \cup \cdots \cup H_{s+1}\right| \\
& \leqslant|I|+\left|H_{1}\right|+\cdots+\left|H_{s+1}\right| \leqslant|I|+(s+1)(\alpha-1),
\end{aligned}
$$

where we abuse the notations $I, \bar{I}$, and $H_{i}$ to refer to the subset of points of $P$ that they contain. This inequality implies that $I$ contains at least $n-(s+1)(\alpha-1)$ points of $P$. This finishes the proof of the theorem except for the part that $P$ is in general position.

Now, assume that $P$ is in general position and $n \geqslant d+3$. By the definition of general position, the number of points of $P$ in a $(d-s)$-dimensional flat is not more than $d-s+1$. Since $I$ is $(d-s)$-dimensional, this implies that

$$
n-(s+1)(\alpha-1) \leqslant d-s+1 .
$$

Notice that $n$ is of the form $k(d+1)+i$ for some integer $k \geqslant 1$ and some $i \in\{0,1, \ldots, d\}$. Moreover, if $i$ is 0 or 1 , then $k \geqslant 2$ because $n \geqslant d+3$. Now we consider two cases depending on whether or not $i$ is 0 . If $i=0$, then $\alpha=k$. In this case, the above inequality simplifies to $k(d-s) \leqslant d-2 s$, which is not possible because $k \geqslant 2$ and $d \geqslant s \geqslant 1$. If $i \in\{1, \ldots, d\}$, then $\alpha=k+1$. In this case, the above inequality simplifies to $(k-1)(d-s)+i \leqslant 1$, which is not possible unless $d=s$ and $i=1$. Thus, for the above inequality to hold we should have $d=s$ and $i=1$. These two assertions imply that $n=k(d+1)+1$, and that $I$ is 0 -dimensional and consists of one point of $P$. Since $C \subseteq I$ and $C$ is not empty, $C$ also consists of one point of $P$. 


\section{References}

[1] O. Aichholzer, T. Hackl, M. Korman, M. J. van Kreveld, M. Löffler, A. Pilz, B. Speckmann, and E. Welzl. Packing plane spanning trees and paths in complete geometric graphs. Information Processing Letters, 124:35-41, 2017. Also in CCCG'14, pages 233-238.

[2] B. Aronov, P. Erdös, W. Goddard, D. J. Kleitman, M. Klugerman, J. Pach, and L. J. Schulman. Crossing families. Combinatorica, 14(2):127-134, 1994. Also in SoCG'91, pages $351-356$.

[3] F. Bernhart and P. C. Kainen. The book thickness of a graph. Journal of Combinatorial Theory, Series B, 27(3):320-331, 1979.

[4] A. Biniaz, P. Bose, A. Maheshwari, and M. H. M. Smid. Packing plane perfect matchings into a point set. Discrete Mathematics \& Theoretical Computer Science, 17(2):119-142, 2015.

[5] P. Bose, F. Hurtado, E. Rivera-Campo, and D. R. Wood. Partitions of complete geometric graphs into plane trees. Computational Geometry: Theory and Applications, 34(2):116-125, 2006.

[6] T. M. Chan. An optimal randomized algorithm for maximum tukey depth. In Proceedings of the 15th Annual ACM-SIAM Symposium on Discrete Algorithms, SODA, pages 430-436, 2004.

[7] H. Edelsbrunner. Algorithms in Combinatorial Geometry. Springer, 1987.

[8] B. Grünbaum. The dimension of intersections of convex sets. Pacific Journal of Mathematics, 12(1):197-202, 1962.

[9] S. Jadhav and A. Mukhopadhyay. Computing a centerpoint of a finite planar set of points in linear time. Discrete \& Computational Geometry, 12:291-312, 1994.

[10] S. Kundu. Bounds on the number of disjoint spanning trees. Journal of Combinatorial Theory, Series B, 17(2):199-203, 1974.

[11] C. St. J. A. Nash-Williams. Edge-disjoint spanning trees of finite graphs. Journal of the London Mathematical Society, 36(1):445-450, 1961.

[12] P. Schnider. Packing plane spanning double stars into complete geometric graphs. In Proceedings of the 32nd European Workshop on Computational Geometry, EuroCG, pages 91-94, 2016.

[13] W. T. Tutte. On the problem of decomposing a graph into $n$ connected factors. Journal of the London Mathematical Society, 36(1):221-230, 1961. 